\section{Research Article Periodontal Science}

Check for updates

\section{OPEN ACCESS}

Received: Sep 17, 2018

Accepted: Jan 30, 2019

*Correspondence:

Sungtae Kim

Department of Periodontology, Dental

Research Institute, Seoul National University

School of Dentistry, 101 Daehak-ro, Jongno-gu,

Seoul 03080, Korea.

E-mail: kst72@snu.ac.kr

Tel: +82-2-2072-4712

Fax: +82-2-744-0051

+Heesuk An and Jung-Tae Lee equally

contributed to this study.

Copyright (C) 2019. Korean Academy of

Periodontology

This is an Open Access article distributed under the terms of the Creative Commons Attribution Non-Commercial License (https:// creativecommons.org/licenses/by-nc/4.0/).

ORCID iDs

Heesuk An (D)

https://orcid.org/0000-0001-5657-8133

Jung-Tae Lee (iD)

https://orcid.org/0000-0001-5383-3004

Seo-Eun Oh (iD)

https://orcid.org/0000-0003-3308-3423

Kyeong-mee Park (D)

https://orcid.org/0000-0002-1301-0959

Kyung-Seok Hu (D)

https://orcid.org/0000-0002-9048-3805

Sungtae Kim (D)

https://orcid.org/0000-0001-6361-4104

Moon-Kyu Chung (iD)

https://orcid.org/0000-0002-5095-4828

\title{
Adjunctive hyperbaric oxygen therapy for irradiated rat calvarial defects
}

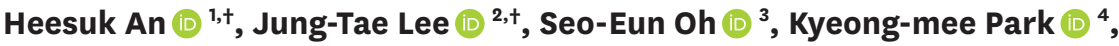 \\ Kyung-Seok Hu (iD ${ }^{5}$, Sungtae Kim (i]) ${ }^{2, *}$, Moon-Kyu Chung (i) ${ }^{1}$
}

'Department of Prosthodontics, Yonsei University College of Dentistry, Seoul, Korea

${ }^{2}$ Department of Periodontology, Dental Research Institute, Seoul National University School of Dentistry, Seoul, Korea

${ }^{3}$ Department of Periodontology, Dental Research Institute, Seoul National University School of Dentistry, Seoul, Korea

${ }^{4}$ Department of Advanced General Dentistry, Yonsei University College of Dentistry, Seoul, Korea ${ }^{5}$ Division in Anatomy and Developmental Biology, Department of Oral Biology, Human Identification Research Institute, BK21 PLUS Project, Yonsei University College of Dentistry, Seoul, Korea

\section{ABSTRACT}

Purpose: The aim of this study was to conduct a histologic evaluation of irradiated calvarial defects in rats 4 weeks after applying fibroblast growth factor-2 (FGF-2) with hyaluronan or biphasic calcium phosphate (BCP) block in the presence or absence of adjunctive hyperbaric oxygen (HBO) therapy.

Methods: Twenty rats were divided into $\mathrm{HBO}$ and non-HBO (NHBO) groups, each of which was divided into FGF-2 and BCP-block subgroups according to the grafted material. Localized radiation with a single 12-Gy dose was applied to the calvaria of rats to simulate radiotherapy. Four weeks after applying this radiation, 2 symmetrical circular defects with a diameter of $6 \mathrm{~mm}$ were created in the parietal bones of each animal. The right-side defect was filled with the materials mentioned above and the left-side defect was not filled (as a control). All defects were covered with a resorbable barrier membrane. During 4 weeks of healing, 1 hour of HBO therapy was applied to the rats in the HBO groups 5 times a week. The rats were then killed, and the calvarial specimens were harvested for radiographic and histologic analyses. Results: New bone formation was greatest in the FGF-2 subgroup, and improvement was not found in the BCP subgroup. HBO seemed to have a minimal effect on new bone formation. There was tendency for more angiogenesis in the $\mathrm{HBO}$ groups than the NHBO groups, but the group with $\mathrm{HBO}$ and FGF-2 did not show significantly better outcomes than the HBOonly group or the NHBO group with FGF-2.

Conclusions: HBO exerted beneficial effects on angiogenesis in calvarial defects of irradiated rats over a 4-week healing period, but it appeared to have minimal effects on bone regeneration. FGF-2 seemed to enhance new bone formation and angiogenesis, but its efficacy appeared to be reduced when HBO was applied.

Keywords: Biphasic calcium phosphate; Fibroblast growth factor-2; Hyperbaric oxygen therapy; Radiotherapy 


\section{Funding}

This research was supported by a grant of the Korea Health Technology R\&D Project through the Korea Health Industry Development Institute, funded by the Ministry of Health \& Welfare, Republic of Korea (grant number: H175C1535)

\section{Author Contributions}

Conceptualization: Sungtae Kim, Moon-Kyu Chung; Formal analysis: Heesuk An, Jung-Tae Lee; Investigation; Heesuk An, Jung-Tae Lee, Seo-Eun Oh, Kyeong-mee Park, Kyung-Seok $\mathrm{Hu}$; Methodology: Sungtae Kim, Moon-Kyu Chung; Project administration: Sungtae Kim, Moon-Kyu Chung; Resources: Seo-Eun Oh, Kyeong-mee Park; Software: Seo-Eun Oh, Kyeong-mee Park; Writing - original draft: Heesuk An, Jung-Tae Lee; Writing - review \& editing: Kyung-Seok Hu, Sungtae Kim, MoonKyu Chung.

\section{Conflict of Interest}

No potential conflict of interest relevant to this article was reported.

\section{INTRODUCTION}

Radiotherapy after oral and maxillofacial cancer surgery often results in unwanted complications, such as osteoradionecrosis due to delayed bone healing associated with damage to osteoprogenitor cells and reduced neovascularization [1,2]. For example, a previous study found that the bone healing capacity decreased by $70.9 \%$ during a 4 -week healing period after irradiation at bony sites prepared for dental implants in rabbit tibiae [3]. Impaired bone healing, as manifested by woven bone, immature bone marrow, and decreased bone mineral density, has also been reported in irradiated calvarial defects in rats [4]. Furthermore, the bone healing capacity decreases markedly in patients who receive radiotherapy for oral and maxillofacial cancers, which makes the rehabilitation procedure after cancer surgery more challenging [3].

Hyperbaric oxygen ( $\mathrm{HBO}$ ) therapy is regarded a possible adjunctive therapy for the management of irradiated bones whose healing or regenerative capacity decreases due to hypocellularity, hypovascularity, and hypoxia [5]. HBO therapy involves the inhalation of air that contains high amounts of oxygen to permit its systemic diffusion and delivery [6]. This therapy is usually performed in a chamber with $100 \%$ oxygen at an absolute atmospheric pressure between 2.0 and 2.5 atmospheres absolute (ATA). The volume of oxygen combined with hemoglobin in the blood is generally approximately $20 \%$, but the volume of oxygen dissolved in the plasma increases with pressure [6]. The partial pressure of oxygen is normally $5-15 \mathrm{mmHg}$ in irradiated tissues, and increases to 20-35 mmHg after HBO therapy [7]. Inhalation of oxygen at $>1$ ATA promotes the production of reactive oxygen species, which act as signaling molecules for various growth factors, cytokines, and hormones [8]. HBO-mediated oxidative stress stimulates the differentiation of circulating stem/progenitor cells and growth of new blood vessels by local endothelial cells [8]. Despite the effectiveness of HBO therapy in irradiated bones, its effects remain controversial. In animal studies, HBO therapy has been found to promote bone formation in normal bone and angiogenesis in irradiated bone $[9,10]$. However, other studies have argued that there is limited evidence to support any benefits of this therapy [11,12]. A randomized, placebo-controlled trial showed that HBO therapy was ineffective for treating mandibular radionecrosis [13]. Several side effects, such as reversible myopia and barotrauma, have also been reported [14].

Synthetic bone substitute materials are occasionally used for bone regeneration. Biphasic calcium phosphate (BCP), a type of synthetic bone substitute, is composed of hydroxyapatite and $\beta$-tricalcium phosphate. Previous studies have produced favorable results with BCP $[15,16]$. However, BCP lacks osteoinductive capacity, which is one of the drawbacks of using the currently available synthetic bone substitutes. Various attempts have recently been made to enhance the osteogenic potential of these synthetic bone substitutes. One method involves coating BCP with peptides such as bone morphogenetic protein, oligopeptide, or epigallocatechin-3-gallate, which can produce favorable results [17-19]. Another drawback of BCP bone substitute is that it is available in a particulate form, which prevents it from being properly maintained in defects that lack bony walls. A new type of $\mathrm{BCP}$ block has been fabricated using collagen to prevent the dissipation of BCP particles. This BCP block has excellent formability and can fit into the defects more easily; however, the added collagen might delay bone formation because it first needs to be resorbed before space becomes available for the new bone to form $[20,21]$.

Growth factors have significant effects on osteoblast behavior and have thus been widely used for bone regeneration. Fibroblast growth factor-2 (FGF-2) is an effective angiogenic 
and osteogenic growth factor [22]. It has been shown to induce angiogenesis and new bone formation in calvarial defects in rats and is considered to contribute to wound healing by stimulating the proliferation of mesenchymal cells [22]. It is effective both in normal and in irradiated bone [23]. Hyaluronan (HA), which is used as a carrier for FGF-2, is present throughout the body in the extracellular matrix as a naturally occurring biodegradable polymer responsible for the structural properties of tissues [24]. In a previous study, a viscous gel composed of FGF-2 and HA was introduced via injection into fractured bones as a novel treatment [25]. A single administration of this formulated gel in the fibulae of baboons with an experimentally created bilateral 1-mm gap defect accelerated fracture healing, providing evidence for increased callus formation and physical solidity relative to the untreated sites [25].

In light of the abovementioned findings, the aim of this study was to histologically evaluate irradiated rat calvarial defects at 4 weeks after applying FGF-2 or BCP block, with or without adjunctive $\mathrm{HBO}$ therapy.

\section{MATERIALS AND METHODS}

\section{Experimental animals and materials}

The study was carried out on 20 male Sprague-Dawley rats (body weight, 250-300 g, 4 weeks old) and approved by the Institutional Animal Care and Use committee of Yonsei Medical Center, Seoul, Korea (2012-0141-1). All experimental procedures were conducted according to the guidelines for animal experiments of Yonsei University, College of Dentistry.

One of the graft materials was a gel containing 0.07\% FGF-2 (Genoss, Suwon, Korea) with $2 \%$ HA (Genoss), and the other was BCP block (OsteonTM II collagen ${ }^{\circledR}$, Genoss) composed of synthetic bone graft and bovine type I collagen. The BCP block was cut to the dimensions of the defect, with a diameter of $6 \mathrm{~mm}$ and a height of $1.5 \mathrm{~mm}$. A resorbable barrier membrane (HA collagen membrane ${ }^{\circledast}$, Genoss) was used bilaterally to prevent the defect from collapsing.

The rats were divided into $\mathrm{HBO}$ and non-HBO (NHBO) groups, which were each further divided into 2 subgroups: 1) FGF-2 with HA and 2) BCP block. Localized radiation with a single 12-Gy dose was applied to each rat calvarium. Four weeks after applying the radiation, 2 symmetrical circular defects with a diameter of $6 \mathrm{~mm}$ were created in the parietal bones of each animal. The right-side defect was filled with FGF-2 and HA or BCP block, and the leftside defect was not filled (as a control). The study groups can be summarized as follows:

$$
\text { HBO groups: }
$$

FGF-2 with HA (HBO-FGF-2 group; $\mathrm{n}=5$ )

BCP block (HBO-BCP group; $\mathrm{n}=5$ )

Control (HBO-control group; $\mathrm{n}=10$ )

\section{NHBO groups:}

FGF-2 with HA (NHBO-FGF-2 group; n=5)

BCP block (NHBO-BCP group; $n=5$ )

Control (NHBO-control group; $\mathrm{n}=10$ ) 


\section{Protocol for animal irradiation}

General anesthesia was induced with an intraperitoneal injection of an anesthetic cocktail composed of xylazine (Rompun, Bayer Korea, Seoul, Korea) and zolazepam (Zoletil 50, Virbac Laboratories, Carros, France). Rats were immobilized using a customized fixation device. Radiation fields were verified using an external beam simulator (Nucletron, Veenendaal, Netherlands). They received localized radiation with a single 12-Gy dose to the calvaria. The irradiation protocol for the calvaria of the rats was as follows: The sedated rat was placed in the fixation device to keep the animal in a prone and head-first position, with the head immobilized. Using a 2-dimensional simulator (Simulix Evolution, Nucletron), the position of the calvarium in the irradiation field was verified, and the treatment center was marked. The rat and fixation device were transferred to a 6-MV medical linear accelerator (Elekta Synergy, Elekta Oncology Systems, Stockholm, Sweden) that is commonly used for treatment in humans. A $0.5-\mathrm{cm}$ tissue-equivalent bolus was placed on top of the rat's head to ensure electronic equilibrium. Using a field size of $2 \mathrm{~cm} \times 2 \mathrm{~cm}$, a radiation dose of $12 \mathrm{~Gy}$ was delivered in a single application to the calvarium of each rat in 2 parallel-opposed, equallyweighted lateral fields.

\section{Surgical procedure}

Four weeks after radiation, the surgical procedures were conducted under general anesthesia induced by the intraperitoneal injection of an anesthetic mixture of xylazine and zolazepam. The surgical area was shaved, and the skin was washed with $70 \%$ ethanol. An intraperitoneal injection of $0.9 \mathrm{~mL}$ of lidocaine (1:100,000 epinephrine, Yuhan, Seoul, Korea) was administered to control bleeding and provide additional anesthesia. A 20-mm-long midline incision was made in the scalp along the sagittal suture and the flaps were reflected laterally. Two symmetrical circular ( $6 \mathrm{~mm}$ in diameter) transosseous bone defects were then created in both parietal bones with a surgical trephine bur under saline irrigation. The midsagittal suture was not included in the bone defect to avoid damaging the dura mater. The left-side experimental defects were kept empty as a control, and the right-side defects were filled with FGF-2 and HA or BCP block. Resorbable membrane (HA collagen membrane ${ }^{\circledast}$, Genoss) was applied with the aim of minimizing how much the defect collapsed. The skin flaps were repositioned and sutured with nonresorbable suture material. After 10 days, the sutures were removed.

All rats were given free access to food pellets and tap water while they were housed and cared for in the animal experimental laboratory of Yonsei University, College of Dentistry, Seoul, South Korea. The animals were killed after 4 weeks of healing by transcardial perfusion with $4 \%$ paraformaldehyde under general anesthesia. The skin was removed from the calvarium and the calvarial specimens were harvested for histologic, histometric, and micro-computed tomography (CT) analysis. The tissue samples were fixed in 10\% formalin for 24 hours and then decalcified by soaking in formic acid-hydrochloric acid for another 24 hours. They were then embedded in paraffin, with 5 - $\mu$ m-thick sections cut in the transverse plane and stained with hematoxylin and eosin (H\&E). The histologic examination was performed with the aid of a light microscope.

\section{Procedure for hyperbaric oxygen therapy}

During 4 weeks of healing after graft surgery, the rats in the $\mathrm{HBO}$ groups were placed in a hyperbaric chamber and exposed to 100\% oxygen at 2.4 ATA for 1 hour per day 5 times a week [26-28]. During this period, the animals in the NHBO groups were left in their usual housing. The HBO therapy began the day after surgery in a mono-place chamber designed for experimentation. 


\section{Radiographic analysis: micro-CT}

Block sections, including the augmented bony defect and the surrounding bone, were resected and fixed in 10\% neutral-buffered formalin for 10 days. Radiographic images were then obtained using micro-CT (1076, SkyScan, Aartselaar, Belgium) at a resolution of $35 \mu \mathrm{m}$ (achieved using $100 \mathrm{kV}$ and $100 \mu \mathrm{A}$ ). OnDemand3D software (Cybermed, Seoul, Korea) was used to reconstruct the area of interest.

\section{Histologic analysis}

All specimens were decalcified with $10 \%$ EDTA at $4^{\circ} \mathrm{C}$ for 1 month. The decalcified specimens were first embedded in paraffin wax, and then a series of $5-\mu \mathrm{m}$-thick sections was prepared. The sections were stained with H\&E and then observed with the aid of an optical microscope (BX51, Olympus, Tokyo, Japan). The area and length of new bone were measured with an image analysis system (Tomoro Scope Eye, Techsan Digital Imaging, Seoul, Korea) after taking images with a digital camera (OlympusBX50, Olympus) at magnifications of $\times 12.5$ and $\times 50$.

\section{Histometric analysis}

Images of histologic sections were captured with a digital camera (BX50, Olympus) at original magnifications of $\times 12.5$ and $\times 50$. Those images were saved on a computer. Histometric analysis was performed with the aid of image analysis software (version 3.6, Tomoro Scope Eye, Techsan Digital Imaging). The most central section was used to assess defect closure (width) and the percentage of bone formation (Figure 1A).
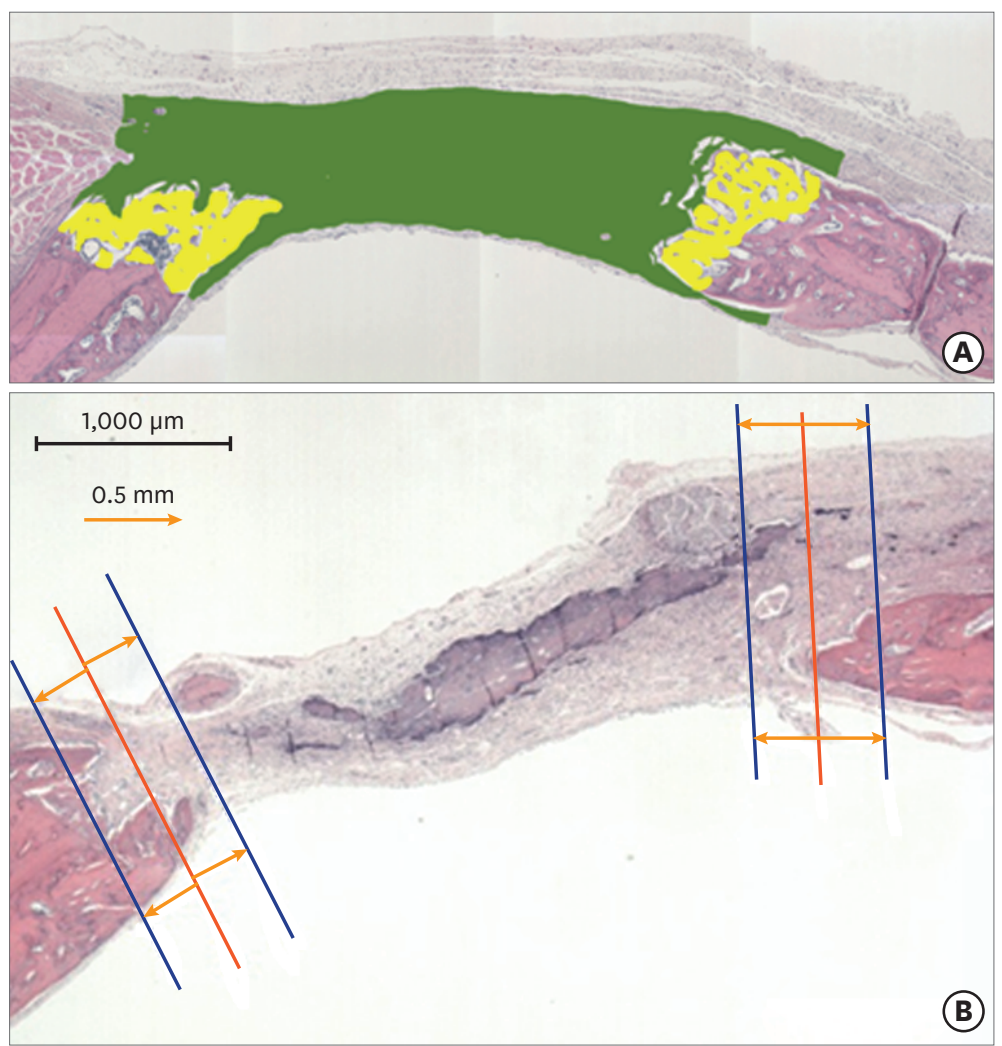

Figure 1. Method of measuring new bone area (A) and length (B) (hematoxylin and eosin). 


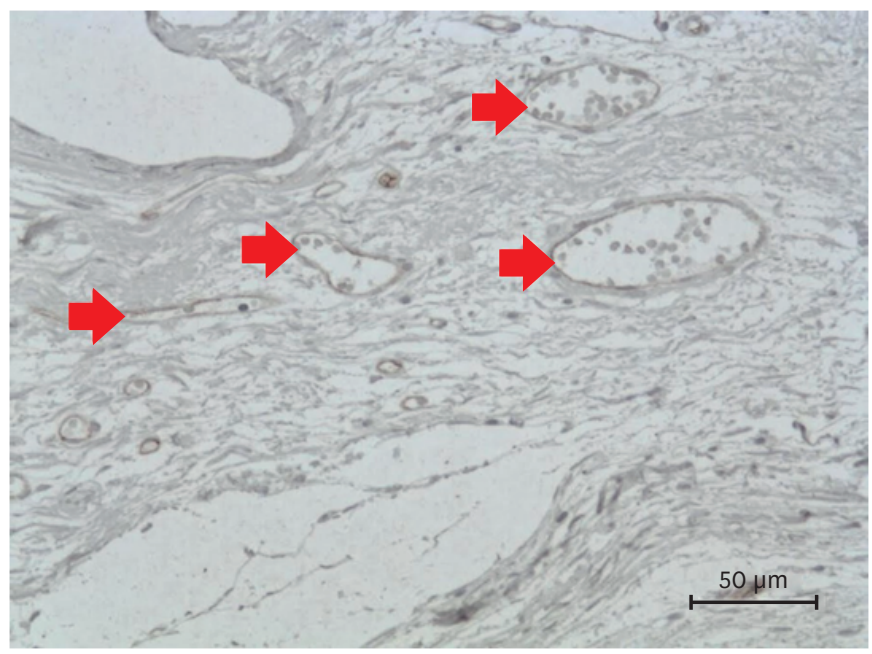

Figure 2. Method of counting blood vessels (diaminobenzidine) - arrowheads.

\section{Immunohistological analysis}

Microvessels were identified using immunohistochemical staining with monoclonal antibodies against endothelial cell adhesion molecule-1 (sc-1506-R, Santa Cruz Biotechnology, Santa Cruz, CA, USA). The sections were incubated for 30 minutes with horseradish peroxidase-labeled antirabbit immunoglobulin antibody (Dako, Glostrup, Denmark) after incubation with the primary antibodies. The sections were then incubated at room temperature for a further 20 minutes after rinsing in Tris-buffered saline, and then stained with diaminobenzidine (DAB) for 15 minutes. An optical microscope was used to evaluate the distribution and localization of stained endothelial cells in the defects. An overview image of the specimens was obtained at a magnification of $\times 200$. DAB staining was evaluated on both defect sides within a 1-mm-wide strip (Figures 1B and 2).

\section{Statistical analysis}

Statistical analyses were performed using SPSS (version 18.0, SPSS, Chicago, IL, USA). The data are presented as mean values \pm standard deviation. To determine whether statistically significant differences existed among the 3 groups, the Kruskal-Wallis test was used. The cutoff for statistical significance was set at $P<0.05$. Three sets of pairs were compared using the Mann-Whitney U test with the Bonferroni correction. $P$ values $<0.017$ were considered to statistical significance for these comparisons.

\section{RESULTS}

\section{Radiographic analysis}

Partial defect closure was found in all groups, and remnants of collagen membrane were found in all defects (Figure 3A-F). The bone substitute was well maintained in the $\mathrm{BCP}$ groups (Figure $3 \mathrm{~B}$ and $\mathrm{E}$ ). New bone formation in the $\mathrm{BCP}$ groups could not be differentiated from BCP particles on micro-CT. The amount of new bone formation around the defect margin was greater in the FGF-2 groups (Figure $3 \mathrm{C}$ and F) than in the control group (Figure $3 \mathrm{~A}$ and $\mathrm{D}$ ). The effect of $\mathrm{HBO}$ on new bone formation could not be evaluated using micro-CT data. 


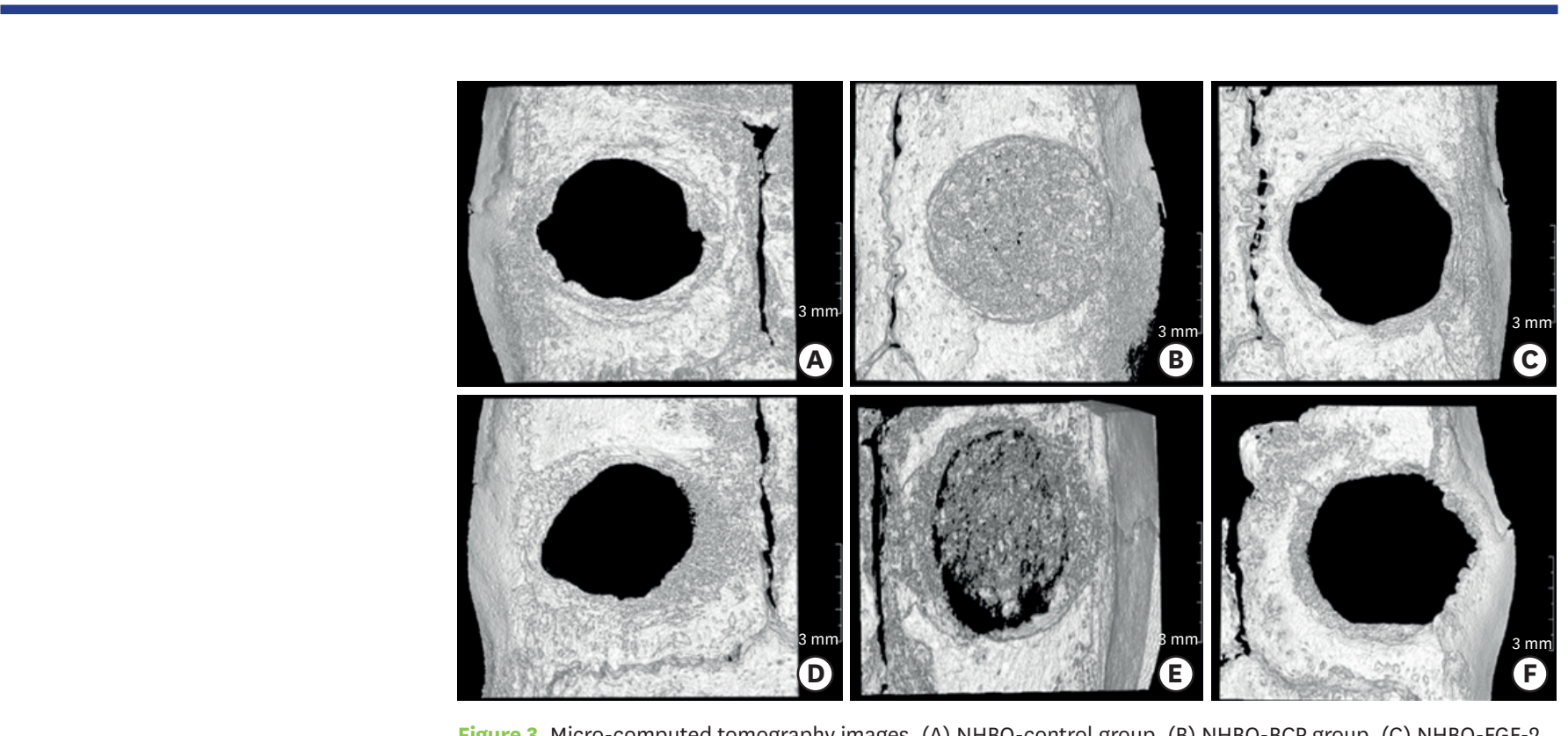

Figure 3. Micro-computed tomography images. (A) NHBO-control group, (B) NHBO-BCP group, (C) NHBO-FGF-2 group, (D) HBO-control group, (E) HBO-BCP group, (F) HBO-FGF-2 group.

NHBO: without adjunctive hyperbaric oxygen treatment, BCP: biphasic calcium phosphate, FGF-2: fibroblast growth factor-2, $\mathrm{HBO}$ : adjunctive hyperbaric oxygen treatment.

\section{Histologic analysis}

After the 4-week healing period, complete defect closure was not observed in any of the calvarial defects. However, there was no sign of inflammation or infection in any of the groups. A limited amount of new bone formation was observed in the control groups, but only around the defect margins. The dimensions of the defect decreased vertically in the control groups because no graft material had been applied (Figures 4A, 4D, 5A, and 5D). The dimensions of the defect were well maintained in the BCP groups. New bone formation was found around

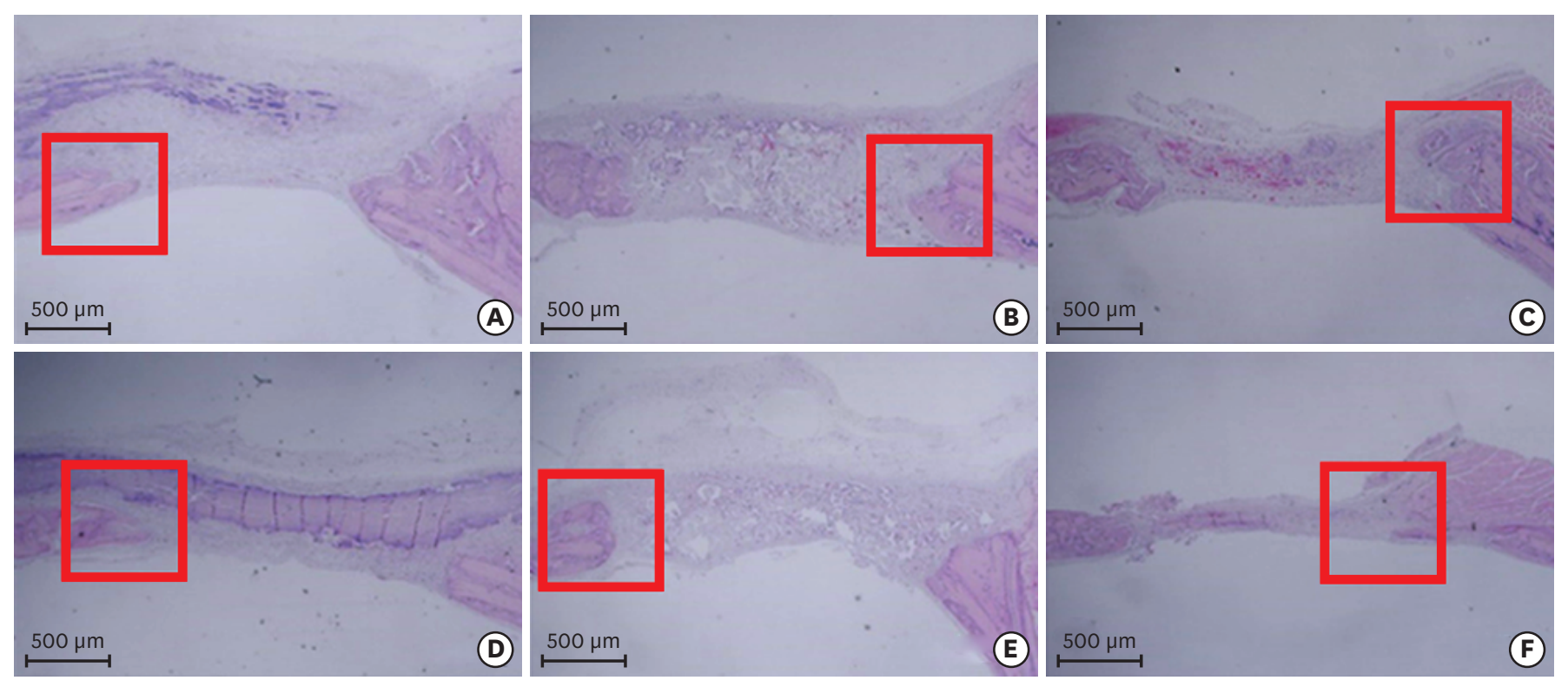

Figure 4. Histological images (hematoxylin and eosin). (A) NHBO-control group, (B) HBO-control group, (C) NHBO-BCP group, (D) HBO-BCP group, (E) NHBOFGF-2 group, (F) HBO-FGF-2 group.

NHBO: without adjunctive hyperbaric oxygen treatment, HBO: adjunctive hyperbaric oxygen treatment, BCP: biphasic calcium phosphate, FGF-2: fibroblast growth factor-2. 

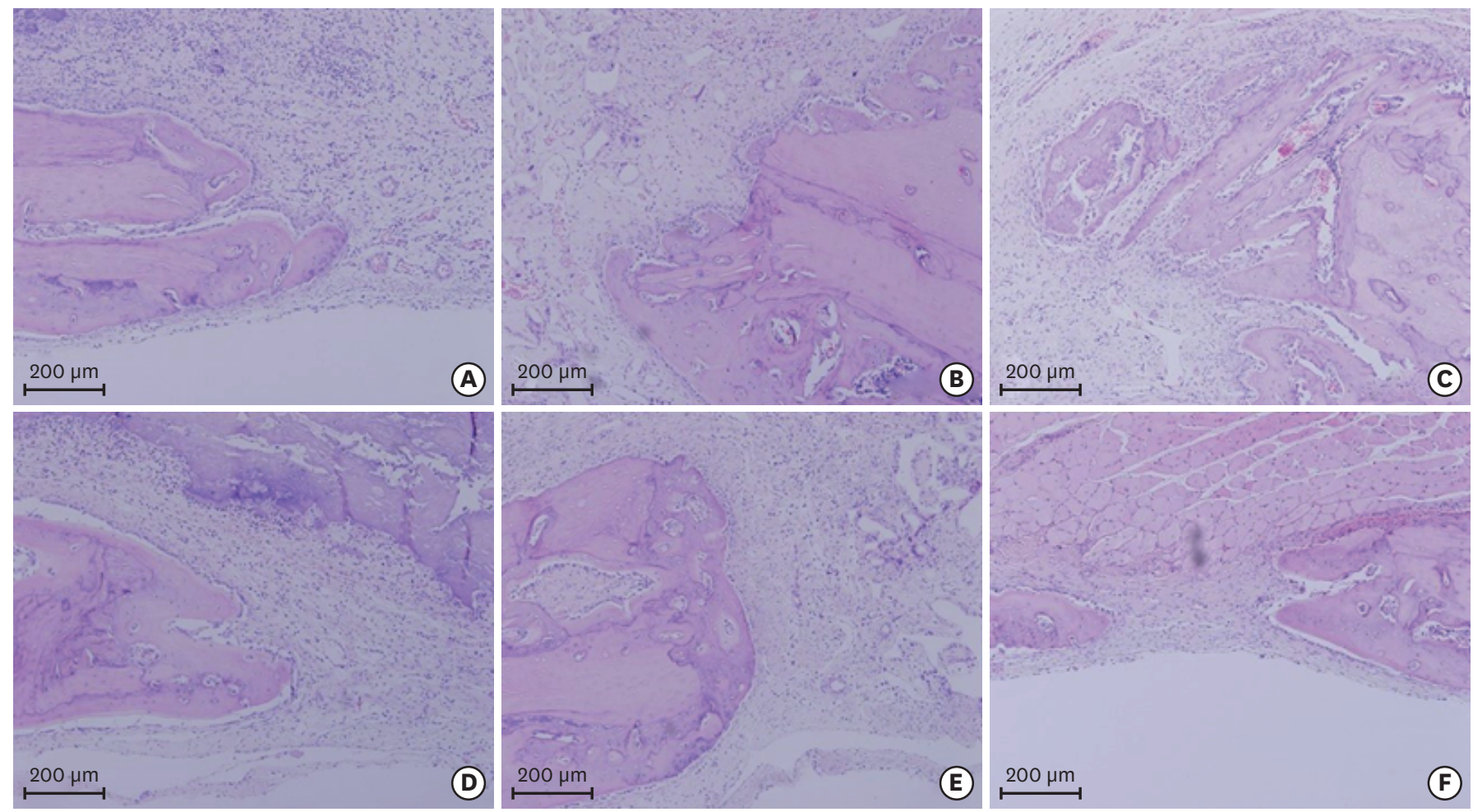

Figure 5. Histological images (hematoxylin and eosin). (A) NHBO-control group, (B) HBO-control group, (C) NHBO-BCP group, (D) HBO-BCP group, (E) NHBOFGF-2 group, (F) HBO-FGF-2 group.

NHBO: without adjunctive hyperbaric oxygen treatment, HBO: adjunctive hyperbaric oxygen treatment, BCP: biphasic calcium phosphate, FGF-2: fibroblast growth factor-2.

the defect margins and $\mathrm{BCP}$ particles (Figures 4B, 4E, 5B, and 5E). New bone was found beside or under the defect margin. The dimensions of the defect also decreased in the FGF-2 groups. New bone formation was found around the defect margins (Figures 4C, 4F, 5C, and 5F). New bone isolated from the defect margin was also found. The amount of new bone formation around the defect margin was greater in the BCP groups than in the control groups.

\section{Histometric analysis}

New bone area

In the HBO group, significant differences were found among all subgroups $(P=0.011)$. In particular, a statistically significant difference was found between the BCP and control groups ( $P=0.001$ ). The results of the NHBO group were similar to those of the HBO group. However, no significant difference was found among subgroups ( $P=0.450)$. In the $\mathrm{HBO}$ and NHBO groups, new bone area was larger in the FGF-2 with HA subgroups than in the other subgroups. The presence of BCP block had an adverse effect on new bone area, while HBO appeared to have a minimal effect on new bone area (Table 1).

\section{New bone length}

In the HBO and NHBO groups, new bone length was also larger in the FGF-2 with HA subgroups than in the other subgroups. There was a statistically significant difference among the subgroups within the NHBO group $(P=0.020)$. The $P$ value for the difference between the $\mathrm{BCP}$ and control subgroups in the NHBO group was close to significant $(P=0.019)$. Similar to new bone area, BCP block seemed to have an adverse effect on new bone length, while HBO had a minimal effect (Table 1 ). 


\begin{tabular}{|c|c|c|c|}
\hline Group & New bone area $\left(\mathrm{mm}^{2}\right)$ & New bone length ( $\mathrm{mm})$ & Blood vessel count (No.) \\
\hline \multicolumn{4}{|l|}{$\mathrm{HBO}$} \\
\hline HBO-FGF-2 $(n=5)$ & $0.36 \pm 0.26$ & $0.93 \pm 0.26$ & $153.2 \pm 50.8$ \\
\hline HBO-BCP $(n=5)$ & $0.17 \pm 0.04$ & $0.58 \pm 0.15$ & $234.0 \pm 41.1$ \\
\hline HBO-control $(n=10)$ & $0.30 \pm 0.06$ & $0.84 \pm 0.30$ & $177.3 \pm 49.8$ \\
\hline$P$ value (FGF-2 vs. BCP vs. control) & $0.011^{\mathrm{a})}$ & 0.068 & $0.048^{a)}$ \\
\hline$P$ value (FGF-2 vs. control) & 0.594 & 0.513 & 0.310 \\
\hline$P$ value (BCP vs. control) & $0.001^{b)}$ & 0.055 & 0.055 \\
\hline$P$ value (FGF-2 vs. BCP) & 0.056 & 0.056 & 0.032 \\
\hline$P$ value (FGF-2 vs. FGF-2 in NHBO) & 0.548 & 0.690 & 0.548 \\
\hline$P$ value (BCP vs. $\mathrm{BCP}$ in $\mathrm{NHBO}$ ) & 1.000 & 0.548 & $0.016^{c)}$ \\
\hline \multicolumn{4}{|l|}{ NHBO } \\
\hline NHBO-FGF-2 $(n=5)$ & $0.34 \pm 0.09$ & $1.00 \pm 0.34$ & $163.2 \pm 43.0$ \\
\hline NHBO-BCP $(n=5)$ & $0.17 \pm 0.11$ & $0.51 \pm 0.18$ & $161.8 \pm 28.1$ \\
\hline NHBO-control $(n=10)$ & $0.29 \pm 0.12$ & $0.81 \pm 0.19$ & $122.3 \pm 27.9$ \\
\hline$P$ value (FGF-2 vs. BCP vs. control) & 0.450 & $0.020^{\mathrm{a})}$ & 0.051 \\
\hline$P$ value (FGF-2 vs. control) & 0.440 & 0.206 & 0.099 \\
\hline$P$ value (BCP vs. control) & 0.099 & 0.019 & 0.028 \\
\hline$P$ value (FGF-2 vs. BCP) & 0.056 & 0.032 & 1.000 \\
\hline
\end{tabular}

Values are presented as mean \pm standard deviation.

HBO: adjunctive hyperbaric oxygen treatment, FGF-2: fibroblast growth factor-2, BCP: biphasic calcium phosphate, NHBO: without adjunctive hyperbaric oxygen treatment.

${ }^{\text {a) }}$ Statistically significant difference $(P<0.05)$ (Kruskal-Wallis test); ${ }^{\text {b) }}$ Statistically significant difference $(P<0.017$, an adjusted significance level using Bonferroni correction); ${ }^{c}$ Statistically significant difference compared to the corresponding parameter in the HBO group $(P<0.05)$.

\section{Immunohistological analysis}

In the HBO group, significant differences were found among the 3 subgroups $(P=0.048)$. The $P$ value for differences among the subgroups in the NHBO group was close to significant $(P=0.051)$. A statistically significant difference was observed between the HBO-BCP subgroup and the NHBO-BCP subgroup $(P=0.016)$. In general, more angiogenesis was found in the HBO groups than in the NHBO groups. However, when FGF-2 with HA was applied together with $\mathrm{HBO}$ therapy, angiogenesis was not improved relative to $\mathrm{HBO}$ alone or the NHBO group with FGF-2 and HA (Table 1).

\section{DISCUSSION}

In this study, FGF-2 with HA was used for the treatment of irradiated calvarial defects in rats because this combination was considered to facilitate both angiogenesis and osteogenesis. A previous study found that FGF-2 contributed to wound healing by stimulating the proliferation of mesenchymal cells and inducing angiogenesis and new bone formation in rat calvarial defects [22]. Another study showed that FGF-2 increased new bone formation in periodontal defects [23]. In this study, rats administered FGF-2 tended to show more new bone and blood vessel formation than the rats in the control groups. Moreover, new bone isolated from the defect margin formed in the FGF-2-administered rats. These results suggest that FGF-2 has the potential to induce new bone formation, although they did not show statistical significance. This most likely occurred because the healing period of 4 weeks was very short for determining differences in bone formation in irradiated calvarial defects. Further studies should be performed to investigate defects under the same conditions for a longer healing period. 
Most reconstructions after resective surgery and radiotherapy are performed on bony defects that do not have bony walls. This clinical situation prevents the bone substitute from being properly maintained in the defect. BCP block, which comprises BCP particles and collagen, can be considered as an ideal substitute because the collagen is resorbed after the BCP particles, and new bone is formed in the space left thereafter. Therefore, new bone formation takes longer when using a BCP block than when using particulate BCP. The healing capacity of the irradiated bone in this study was lower than that observed in previous studies [23]. It seems that 4 weeks was not long enough for the irradiated bony defects to heal. Further studies should be performed to investigate the effect of BCP blocks over a longer healing time. In this study, the increased vascularity found in the BCP block groups can be attributed to collagen resorption because more blood vessels were observed around the bone substitute than around the defect margin. It is not certain whether this healing process would continue until new bone formation around the bone substitute. The smaller new bone area and length in the BCP block groups relative to the FGF-2 groups can also be attributed to delayed new bone formation when using a BCP block. However, different results are expected with increasing healing time (e.g., 8 weeks).

Previous studies have shown that HBO therapy induced greater new bone formation [29-31], whereas other studies have provided limited evidence for this finding [11,12]. In this study, HBO therapy did not exert any beneficial effect on new bone formation. However, the results could have been different if the healing time had been longer than 4 weeks. A previous study found favorable effects of HBO therapy on new bone formation in healing time frames of 6 and 12 weeks [29]. Moreover, different results may be obtained when using different frequencies and durations of HBO therapy. For example, a previous study, in which HBO therapy was administered for 90 minutes per day 5 times a week for 4 weeks, suggested that HBO therapy had favorable effects on new bone formation [30]. Future studies should therefore are needed for evaluating the effects of other $\mathrm{HBO}$ therapy protocols. Increased angiogenesis was found in the HBO groups in the present study. While it is unclear whether this increased vascularity would favorably affect new bone formation, previous findings suggest that increased vascularity is associated with faster healing and more new bone formation [29-31].

There was a tendency for less bone formation when FGF-2 with HA was applied in combination with HBO therapy (i.e., the HBO-FGF-2 group) relative to using FGF-2 with HA without HBO. There was also less angiogenesis in the HBO-FGF-2 group than in the FGF-2 with HA group (i.e., NHBO-FGF-2) or HBO therapy alone group (i.e., NHBO-control). This result is in accordance with that of a previous study [32]. The previous study also showed that FGF-2 was more effective than HBO therapy in improving bone growth after radiation, and that $\mathrm{HBO}$ therapy did not provide any additional beneficial effect to FGF-2 therapy. It was also explained that both modalities exert their effects via similar pathways. The data obtained in our study, together with the findings of previous studies, suggest that applying materials or modalities that exert similar effects simultaneously cannot be recommended. Future studies should consider a longer healing time, different protocols of HBO therapy, and different types of BCP, compared with this study.

Within the limitations of this study, our results suggest that $\mathrm{HBO}$ exerted beneficial effects on angiogenesis, but minimal effects on bone regeneration, in an irradiated rat model. FGF-2 with HA enhanced bone generation and angiogenesis, but its efficacy was reduced when HBO was also applied. Using BCP block did not have any beneficial effect on bone regeneration. 


\section{REFERENCES}

1. Anderson L, Meraw S, Al-Hezaimi K, Wang HL. The influence of radiation therapy on dental implantology. Implant Dent 2013;22:31-8. PUBMED | CROSSREF

2. Granström G, Jacobsson M, Tjellström A. Titanium implants in irradiated tissue: benefits from hyperbaric oxygen. Int J Oral Maxillofac Implants 1992;7:15-25. PUBMED

3. Jacobsson MG, Jönsson AK, Albrektsson TO, Turesson IE. Short- and long-term effects of irradiation on bone regeneration. Plast Reconstr Surg 1985;76:841-50. PUBMED | CROSSREF

4. Hu WW, Ward BB, Wang Z, Krebsbach PH. Bone regeneration in defects compromised by radiotherapy. J Dent Res 2010;89:77-81. PUBMED | CROSSREF

5. Marx RE. Osteoradionecrosis: a new concept of its pathophysiology. J Oral Maxillofac Surg 1983;41:283-8. PUBMED | CROSSREF

6. Löndahl M. Hyperbaric oxygen therapy as adjunctive treatment of diabetic foot ulcers. Med Clin North Am 2013;97:957-80. PUBMED | CROSSREF

7. Marx RE, Ames JR. The use of hyperbaric oxygen therapy in bony reconstruction of the irradiated and tissue-deficient patient. J Oral Maxillofac Surg 1982;40:412-20. PUBMED | CROSSREF

8. Thom SR. Hyperbaric oxygen: its mechanisms and efficacy. Plast Reconstr Surg 2011;127 Suppl 1:131S-141S PUBMED | CROSSREF

9. Sawai T, Niimi A, Takahashi H, Ueda M. Histologic study of the effect of hyperbaric oxygen therapy on autogenous free bone grafts. J Oral Maxillofac Surg 1996;54:975-81. PUBMED | CROSSREF

10. Marx RE, Ehler WJ, Tayapongsak P, Pierce LW. Relationship of oxygen dose to angiogenesis induction in irradiated tissue. Am J Surg 1990;160:519-24. PUBMED | CROSSREF

11. Pitak-Arnnop P, Hemprich A, Dhanuthai K, Pausch NC. A systematic review in 2008 did not show value of hyperbaric oxygen therapy for osteoradionecrosis. J Oral Maxillofac Surg 2010;68:2644-5. PUBMED | CROSSREF

12. Fritz GW, Gunsolley JC, Abubaker O, Laskin DM. Efficacy of pre- and postirradiation hyperbaric oxygen therapy in the prevention of postextraction osteoradionecrosis: a systematic review. J Oral Maxillofac Surg 2010;68:2653-60. PUBMED | CROSSREF

13. Annane D, Depondt J, Aubert P, Villart M, Géhanno P, Gajdos P, et al. Hyperbaric oxygen therapy for radionecrosis of the jaw: a randomized, placebo-controlled, double-blind trial from the ORN96 study group. J Clin Oncol 2004;22:4893-900. PUBMED | CROSSREF

14. Tibbles PM, Edelsberg JS. Hyperbaric-oxygen therapy. N Engl J Med 1996;334:1642-8. PUBMED | CROSSREF

15. Nevins M, Nevins ML, Schupbach P, Kim SW, Lin Z, Kim DM. A prospective, randomized controlled preclinical trial to evaluate different formulations of biphasic calcium phosphate in combination with a hydroxyapatite collagen membrane to reconstruct deficient alveolar ridges. J Oral Implantol 2013;39:133-9. PUBMED | CROSSREF

16. Mangano C, Perrotti V, Shibli JA, Mangano F, Ricci L, Piattelli A, et al. Maxillary sinus grafting with biphasic calcium phosphate ceramics: clinical and histologic evaluation in man. Int J Oral Maxillofac Implants 2013;28:51-6. PUBMED | CROSSREF

17. Choi H, Park NJ, Jamiyandorj O, Choi KH, Hong MH, Oh S, et al. Improvement of osteogenic potential of biphasic calcium phosphate bone substitute coated with two concentrations of expressed recombinant human bone morphogenetic protein 2. J Periodontal Implant Sci 2012;42:119-26. PUBMED | CROSSREF

18. Choi H, Park NJ, Jamiyandorj O, Hong MH, Oh S, Park YB, et al. Improvement of osteogenic potential of biphasic calcium phosphate bone substitute coated with synthetic cell binding peptide sequences. J Periodontal Implant Sci 2012;42:166-72. PUBMED | CROSSREF 
19. Shin YS, Seo JY, Oh SH, Kim JH, Kim ST, Park YB, et al. The effects of ErhBMP-2-/EGCG-coated BCP bone substitute on dehiscence around dental implants in dogs. Oral Dis 2014;20:281-7. PUBMED | CROSSREF

20. Clergeau LP, Danan M, Clergeau-Guérithault S, Brion M. Healing response to anorganic bone implantation in periodontal intrabony defects in dogs. Part I. Bone regeneration. A microradiographic study. J Periodontol 1996;67:140-9. PUBMED | CROSSREF

21. Hartman GA, Arnold RM, Mills MP, Cochran DL, Mellonig JT. Clinical and histologic evaluation of anorganic bovine bone collagen with or without a collagen barrier. Int J Periodontics Restorative Dent 2004;24:127-35. PUBMED

22. Kigami R, Sato S, Tsuchiya N, Yoshimakai T, Arai Y, Ito K. FGF-2 angiogenesis in bone regeneration within critical-sized bone defects in rat calvaria. Implant Dent 2013;22:422-7. PUBMED | CROSSREF

23. Anzai J, Kitamura M, Nozaki T, Nagayasu T, Terashima A, Asano T, et al. Effects of concomitant use of fibroblast growth factor (FGF)-2 with beta-tricalcium phosphate ( $\beta$-TCP) on the beagle dog 1-wall periodontal defect model. Biochem Biophys Res Commun 2010;403:345-50. PUBMED | CROSSREF

24. Fakhari A, Berkland C. Applications and emerging trends of hyaluronic acid in tissue engineering, as a dermal filler and in osteoarthritis treatment. Acta Biomater 2013;9:7081-92. PUBMED | CROSSREF

25. Radomsky ML, Aufdemorte TB, Swain LD, Fox WC, Spiro RC, Poser JW. Novel formulation of fibroblast growth factor-2 in a hyaluronan gel accelerates fracture healing in nonhuman primates. J Orthop Res 1999;17:607-14. PUBMED | CROSSREF

26. Kawada S, Wada E, Matsuda R, Ishii N. Hyperbaric hyperoxia accelerates fracture healing in mice. PLoS One 2013;8:e72603. PUBMED | CROSSREF

27. Sawai T, Niimi A, Takahashi H, Ueda M. Histologic study of the effect of hyperbaric oxygen therapy on autogenous free bone grafts. J Oral Maxillofac Surg 1996;54:975-81. PUBMED | CROSSREF

28. Sirin Y, Olgac V, Dogru-Abbasoglu S, Tapul L, Aktas S, Soley S. The influence of hyperbaric oxygen treatment on the healing of experimental defects filled with different bone graft substitutes. Int J Med Sci 2011;8:114-25. PUBMED | CROSSREF

29. Jan AM, Sándor GK, Iera D, Mhawi A, Peel S, Evans AW, et al. Hyperbaric oxygen results in an increase in rabbit calvarial critical sized defects. Oral Surg Oral Med Oral Pathol Oral Radiol Endod 2006;101:144-9. PUBMED | CROSSREF

30. Jan A, Sándor GK, Brkovic BB, Peel S, Evans AW, Clokie CM. Effect of hyperbaric oxygen on grafted and nongrafted calvarial critical-sized defects. Oral Surg Oral Med Oral Pathol Oral Radiol Endod 2009;107:157-63. PUBMED | CROSSREF

31. Jan A, Sándor GK, Brkovic BB, Peel S, Kim YD, Xiao WZ, et al. Effect of hyperbaric oxygen on demineralized bone matrix and biphasic calcium phosphate bone substitutes. Oral Surg Oral Med Oral Pathol Oral Radiol Endod 2010;109:59-66. PUBMED | CROSSREF

32. Wang X, Ding I, Xie H, Wu T, Wersto N, Huang K, et al. Hyperbaric oxygen and basic fibroblast growth factor promote growth of irradiated bone. Int J Radiat Oncol Biol Phys 1998;40:189-96. PUBMED | CROSSREF 